\title{
High-Dose Rifapentine with Moxifloxacin for Pulmonary Tuberculosis
}

\begin{abstract}
Amina Jindani, F.R.C.P., Thomas S. Harrison, F.R.C.P., Andrew J. Nunn, M.Sc., Patrick P.J. Phillips, Ph.D., Gavin J. Churchyard, Ph.D., Salome Charalambous, Ph.D., Mark Hatherill, M.D., Hennie Geldenhuys, M.B., Ch.B., Helen M. Mcllleron, Ph.D., Simbarashe P. Zvada, M.Phil., Stanley Mungofa, M.P.H., Nasir A. Shah, M.B., B.S., Simukai Zizhou, M.B., Ch.B., Lloyd Magweta, M.B., Ch.B., James Shepherd, Ph.D., Sambayawo Nyirenda, M.D., Janneke H. van Dijk, Ph.D., Heather E. Clouting, M.Sc., David Coleman, M.Sc., Anna L.E. Bateson, Ph.D., Timothy D. McHugh, Ph.D., Philip D. Butcher, Ph.D., and Denny A. Mitchison, F.R.C.P., for the RIFAQUIN Trial Team*
\end{abstract}

A BSTRACT

\section{BACKGROUND}

Tuberculosis regimens that are shorter and simpler than the current 6-month daily regimen are needed.

\section{METHODS}

We randomly assigned patients with newly diagnosed, smear-positive, drug-sensitive tuberculosis to one of three regimens: a control regimen that included 2 months of ethambutol, isoniazid, rifampicin, and pyrazinamide administered daily followed by 4 months of daily isoniazid and rifampicin; a 4-month regimen in which the isoniazid in the control regimen was replaced by moxifloxacin administered daily for 2 months followed by moxifloxacin and $900 \mathrm{mg}$ of rifapentine administered twice weekly for 2 months; or a 6-month regimen in which isoniazid was replaced by daily moxifloxacin for 2 months followed by one weekly dose of both moxifloxacin and $1200 \mathrm{mg}$ of rifapentine for 4 months. Sputum specimens were examined on microscopy and after culture at regular intervals. The primary end point was a composite treatment failure and relapse, with noninferiority based on a margin of 6 percentage points and $90 \%$ confidence intervals.

\section{RESULTS}

We enrolled a total of 827 patients from South Africa, Zimbabwe, Botswana, and Zambia; $28 \%$ of patients were coinfected with the human immunodefiency virus. In the per-protocol analysis, the proportion of patients with an unfavorable response was $4.9 \%$ in the control group, 3.2\% in the 6-month group (adjusted difference from control, -1.8 percentage points; $90 \%$ confidence interval [CI], -6.1 to 2.4 ), and $18.2 \%$ in the 4-month group (adjusted difference from control, 13.6 percentage points; $90 \% \mathrm{CI}, 8.1$ to 19.1). In the modified intention-to-treat analysis these proportions were $14.4 \%$ in the control group, $13.7 \%$ in the 6-month group (adjusted difference from control, 0.4 percentage points; $90 \% \mathrm{CI},-4.7$ to 5.6 ), and $26.9 \%$ in the 4-month group (adjusted difference from control, 13.1 percentage points; $90 \%$ CI, 6.8 to 19.4).

\section{CONCLUSIONS}

The 6-month regimen that included weekly administration of high-dose rifapentine and moxifloxacin was as effective as the control regimen. The 4-month regimen was not noninferior to the control regimen. (Funded by the European and Developing Countries Clinical Trials Partnership and the Wellcome Trust; RIFAQUIN Current Controlled Trials number, ISRCTN44153044.)
From St. George's, University of London (A.J., D.A.M., T.S.H., D.C., P.D.B.), Medical Research Council Clinical Trials Unit at University College London (A.J.N., P.P.J.P., H.E.C.), and University College London Centre for Clinical Biology, University College London (A.L.E.B., T.D.M.), London; Aurum Institute (G.J.C., S.C.) and School of Public Health, University of the Witwatersrand (G.J.C.), Johannesburg, and South African Tuberculosis Vaccine Initiative, Institute of Infectious Disease and Molecular Medicine and School of Child and Adolescent Health (M.H., H.G.) and Division of Clinical Pharmacology, Department of Medicine (H.M.M., S.P.Z.), University of Cape Town, Cape Town - all in South Africa; Harare City Health Department, Harare (S.M., N.A.S.S.), and Medical Directorate of Mashonaland East, Marondera (S.Z., L.M.) - both in Zimbabwe; CDC, Gaborone, Botswana (J.S., S.N.); and Macha Research Trust, Macha, Zambia (J.H.D.). Address reprint requests to Dr. Jindani at the Institute for Infection and Immunity, St. George's, University of London, Cranmer Terrace, London SWI7 ORE, United Kingdom, or at ajindani@ sgul.ac.uk.

*A complete list of the investigators in the RIFAQUIN Trial is available in the Supplementary Appendix, available at NEJM.org.

N EnglJ Med 2014;371:1599-608. DOI: 10.1056/NEJMoa1314210

Copyright (@) 2014 Massachusetts Medical Society. 
$\mathrm{E}$ FFECTIVE 6-MONTH CHEMOTHERAPY regimens for the treatment of pulmonary tuberculosis have been evaluated in numerous controlled trials. When adequately administered, they are capable of achieving relapse-free cure rates of $95 \%$ or more in patients infected by drug-sensitive organisms. ${ }^{1,2}$ However, there is a need to investigate regimens that could improve operational cure rates by shortening or simplifying treatment. Previous attempts in which intermittent dosing of rifapentine was used $^{3}$ have resulted in unacceptable relapse rates s $^{4,5}$ and, in patients coinfected with the human immunodeficiency virus (HIV), acquired resistance to rifamycin. ${ }^{6}$ Studies in mice suggest that combining high-dose rifapentine with moxifloxacin may improve cure rates. ${ }^{7,8}$ The objectives of this trial were to assess whether regimens of 6 months' or 4 months' duration that included a continuation phase of intermittent treatment with rifapentine and moxifloxacin were not inferior to the standard 6-month regimen based on daily dosing of isoniazid and rifampicin and were able to prevent acquired resistance to rifamycin in relapsing patients who were coinfected with HIV.

METHODS

\section{STUDY OVERSIGHT}

This international, multicenter, randomized, controlled trial was carried out within the framework of the International Consortium for Trials of Chemotherapeutic Agents in Tuberculosis (INTERTB). The trial was sponsored and implemented by St. George's, University of London, together with the Medical Research Council Clinical Trials Unit (MRC CTU) at University College London and six institutions in southern Africa. The study protocol (available with the full text of this article at NEJM .org) was reviewed and approved by the ethics review committee at St. George's by medical ethics and regulatory committees representing each of the participating countries, and by the institutional review board of the Centers for Disease Control and Prevention operating in Botswana. An independent data and safety monitoring committee reviewed study data at intervals of approximately 6 months throughout the study.

\section{PATIENTS}

To participate in the trial, patients had to provide written informed consent, to be older than 18 years of age, to weigh $35 \mathrm{~kg}$ or more, to provide two sputum samples that were positive for tubercle bacilli on direct smear microscopy, to have had less than a month of previous anti-tuberculosis chemotherapy, and to have a firm home address that was readily accessible for visiting. Participants were also expected to survive the initial weeks of treatment. Patients coinfected with HIV who required antiretroviral therapy (ART) at diagnosis were initially ineligible; as the trial progressed, persons starting ART at screening were deemed eligible. Persons with a CD4 cell count of less than 200 per cubic millimeter were initially ineligible, but this number was subsequently amended to 150 per cubic millimeter. ${ }^{3}$

To minimize the risk of the development of multidrug or polydrug resistance and of relapses caused by acquired resistance to rifamycin, patients who had resistance to isoniazid, rifampicin, or moxifloxacin were excluded. In most instances this determination could not be made until after randomization. A complete list of eligibility criteria is provided in the Supplementary Appendix, available at NEJM.org.

After counseling, patients were tested for HIV. The result was disclosed to the patient unless otherwise requested, except in Botswana, where disclosure is mandatory. Post-test counseling was given to all persons who were tested, and those who were infected with HIV were given clinical care within their health service, in accordance with local practice. Chest radiographs were obtained at baseline and evaluated by an experienced assessor to determine the radiographic extent of disease and the presence or absence of cavitation. ${ }^{9,10}$

\section{STUDY DESIGN}

A randomized allocation sequence was generated for each study center with the use of blocks of varying size by an independent statistician based at the MRC CTU. Apart from the statisticians reporting to the data and safety monitoring committee, the staff at St. George's and at the MRC CTU were unaware of treatment assignment except when a lack of awareness would have been unethical (e.g., in some discussions of serious adverse events). Participating laboratories were unaware of treatment assignment throughout the study.

Eligible patients who had provided written informed consent were randomly assigned in a 
ratio of 1:1:1 to one of three regimens: a control regimen, which consisted of 6 months of isoniazid and rifampicin administered daily, supplemented by ethambutol and pyrazinamide in the first 2 months; a 4-month regimen, which consisted of 2 months of ethambutol, moxifloxacin (400 mg), rifampicin, and pyrazinamide administered daily, followed by 2 months of moxifloxacin and rifapentine $(900 \mathrm{mg})$ administered twice weekly; and a 6-month regimen, which consisted of 2 months of ethambutol, moxifloxacin (400 mg), rifampicin, and pyrazinamide administered daily, followed by 4 months of moxifloxacin and rifapentine $(1200 \mathrm{mg})$ administered weekly. All drugs were administered at standard doses $^{11}$ unless otherwise stated; further details are provided in the Supplementary Appendix. Some of the trial medications were donated by Sanofi, Genus Pharmaceuticals, and Sandoz, and a representative of Sanofi was a nonvoting observer at meetings of the steering committee, but none of these companies had any role in the study design, data accrual, data analysis, or manuscript preparation. Because rifapentine absorption is improved when administered with a meal, ${ }^{12}$ each dose of rifapentine was preceded by a meal of two hard-boiled eggs and bread. Treatment was directly observed at the health facility except during the daily continuation phase of the control regimen, during which treatment was supervised by a relative or another person.

Under the conditions of the trial, the standard 6-month regimen has a $95 \%$ or higher success rate. ${ }^{1,2}$ For this reason, the study was designed to determine whether either of the experimental regimens was not inferior to the control regimen when a margin of noninferiority of 6 percentage points was used. The target sample size was 1095; it was calculated with the use of a two-sided $90 \%$ confidence interval to achieve $80 \%$ power. Details of the calculations are provided in the Supplementary Appendix.

\section{EFFICACY AND SAFETY}

Two sputum samples were collected for smears and culture before treatment, and one sample was collected monthly for 12 months and then again at 15 months and 18 months. Samples from centers in Zimbabwe were cultured on LöwensteinJensen solid medium and samples from centers in South Africa and Botswana were cultured in liquid medium in a Mycobacteria Growth Indica- tor Tube. Cultures in Zambia were done on both media. Adverse events were graded for severity with the use of criteria from the National Institute of Allergy and Infectious Diseases, Division of AIDS, after a minor modification..$^{13}$ Most patients (86\%) were followed for 18 months from randomization. However, patients in South Africa and Botswana who underwent randomization in the final 6-month period of enrollment had a reduced follow-up period (either 12 months or 15 months) to permit prolongation of the enrollment period (see the Supplementary Appendix).

The primary efficacy end point was the proportion of patients with an unfavorable outcome, defined as any of the following outcomes: a restart or change of treatment for any reason other than making up for missed doses or becoming pregnant, a positive culture from samples obtained at the most recent follow-up visit, death before the end of scheduled treatment for reasons other than violence or trauma, death after the end of treatment with evidence that confirmed or suggested possible treatment failure or relapse of their tuberculosis, and failure to complete treatment without a negative culture result at the end of the scheduled follow-up period. Relapse after completion of treatment was defined as two positive cultures within a period of 4 months without an intervening negative culture (as in earlier tuberculosis trials ${ }^{2}$ ) and without genotypic evidence of reinfection. A favorable outcome was defined as having a negative culture at the scheduled end of follow-up and not having had an outcome that had already been classified as unfavorable. This definition is consistent with definitions used in several other phase 3 tuberculosis trials. ${ }^{1,14,15}$ More detail is provided in the Supplementary Appendix.

For all treatment failures and relapses, one culture of a sputum sample obtained before treatment and one culture of a sputum sample obtained after treatment failure or relapse were stored at $-20^{\circ} \mathrm{C}$ and the samples were sent to the Department of Medical Microbiology at St. George's for culture and susceptibility testing. Heat-killed organisms were transferred to the Centre for Clinical Microbiology, University College London, for DNA extraction and genotyping by means of mycobacterial interspersed repetitive unit-variablenumber tandem repeats (MIRU-VNTRs). ${ }^{16}$ Patients with a reinfection that was defined as a difference of at least two MIRU loci were excluded from the analysis. 
At each study center, two designated dataentry persons entered case-report forms into a bespoke database developed by the MRC CTU. Data files were transmitted electronically, at regular intervals, to the MRC CTU.

\section{STATISTICAL ANALYSIS}

In accordance with the standard approach to the analysis of noninferiority trials, ${ }^{17}$ the analysis was conducted with both per-protocol and modified intention-to-treat populations. Noninferiority must be shown on both analyses to declare that either regimen is noninferior. The absolute difference in the proportion of unfavorable outcomes was calculated by means of a generalized linear model with an identity-link function. Noninferiority was assessed with the use of the upper bound of the two-sided 90\% Wald confidence interval (which is equivalent to a one-sided significance of $5 \%$ ), and the results were adjusted for study center. One-sided tests are appropriate for noninferiority trials ${ }^{18}$ and a one-sided significance level of $5 \%$ has been used in previous noninferiority trials involving tuberculosis. ${ }^{1,14}$ No adjustment was made for multiple comparisons since each regimen was designed to address different questions. This approach is consistent with expert opinion. ${ }^{19-22}$

Prespecified subgroup analyses were conducted according to HIV status and study center, but results are reported only for analyses made according to HIV status because no heterogeneity was found in analyses conducted according to center. The analysis of culture results at 2 months was limited to participants for whom sputum samples were collected between 6 and 10 weeks after randomization. If more than one culture result was available during this time period, a positive result took precedence over a negative result. All analyses were conducted with the use of STATA software, version 12.1 (Stata). Full details of the analysis strategy are in the Supplementary Appendix.

\section{RESULTS}

\section{STUDY POPULATION}

Between August 15, 2008, and August 1, 2011, a total of 827 patients were enrolled at centers in Worcester (209) and Johannesburg (255), South Africa, in Harare (203) and Marondera (89), Zimbabwe, in Francistown, Botswana (56), and in Macha, Zambia (15). A total of 97 late screening failures were excluded from the primary efficacy analysis; in the majority of these cases, cultures obtained at baseline were either not positive or showed the presence of drug-resistant strains. A total of 593 and 514 patients were included in the modified intention-to-treat and per-protocol analyses, respectively (see the Consolidated Standards for the Reporting of Trials [CONSORT] diagram in Fig. 1 for details on reasons for study exclusion). The baseline characteristics of participants were similar across all three regimens (Table 1).

\section{EFFICACY}

The proportion of patients with unfavorable outcomes among those receiving the control regimen, the 4-month regimen, and the 6-month regimen was $4.9 \%, 18.2 \%$, and $3.2 \%$, respectively, in the per-protocol analysis, and $14.4 \%, 26.9 \%$ and $13.7 \%$, respectively, in the modified intention-to-treat analysis (Table 2). The site-adjusted difference in the proportion of unfavorable outcomes between patients receiving the 4-month regimen and those receiving the control regimen was 13.1 percentage points $(90 \%$ confidence interval [CI], 6.8 to 19.4) in the modified intention-to-treat analysis and $13.6 \%(90 \% \mathrm{CI}, 8.1$ to 19.1) in the per-protocol analysis. In both analyses, the 4-month regimen was inferior to the control regimen, since the lower bound of the $90 \%$ confidence interval was greater than 6 percentage points, although identifying a regimen as inferior was not a prespecified objective of the trial.

The site-adjusted difference in the proportion of unfavorable outcomes between the 6-month regimen and the control regimen was 0.4 percentage points $(90 \% \mathrm{CI},-4.7$ to 5.6$)$ in the modified intention-to-treat analysis and -1.8 percentage points ( $90 \% \mathrm{CI},-6.1$ to 2.4 ) in the per-protocol analysis. In both analyses, the upper bound of the prespecified primary $90 \%$ confidence intervals was less than the margin of noninferiority of 6 percentage points, showing that the 6 -month regimen was noninferior to control regimen when a one-sided significance level of $5 \%$ was used. Although the stricter $95 \%$ confidence interval for the per-protocol analysis gives a consistent interpretation, the $95 \%$ confidence interval for the modified intention-to-treat analysis does not show noninferiority (Table 2). Testing the one-sided null hypothesis that this difference was greater or equal to 6 percentage points produced a $\mathrm{P}$ value of 0.04 for the modified intention-to-treat analysis and a $\mathrm{P}$ value of 0.001 


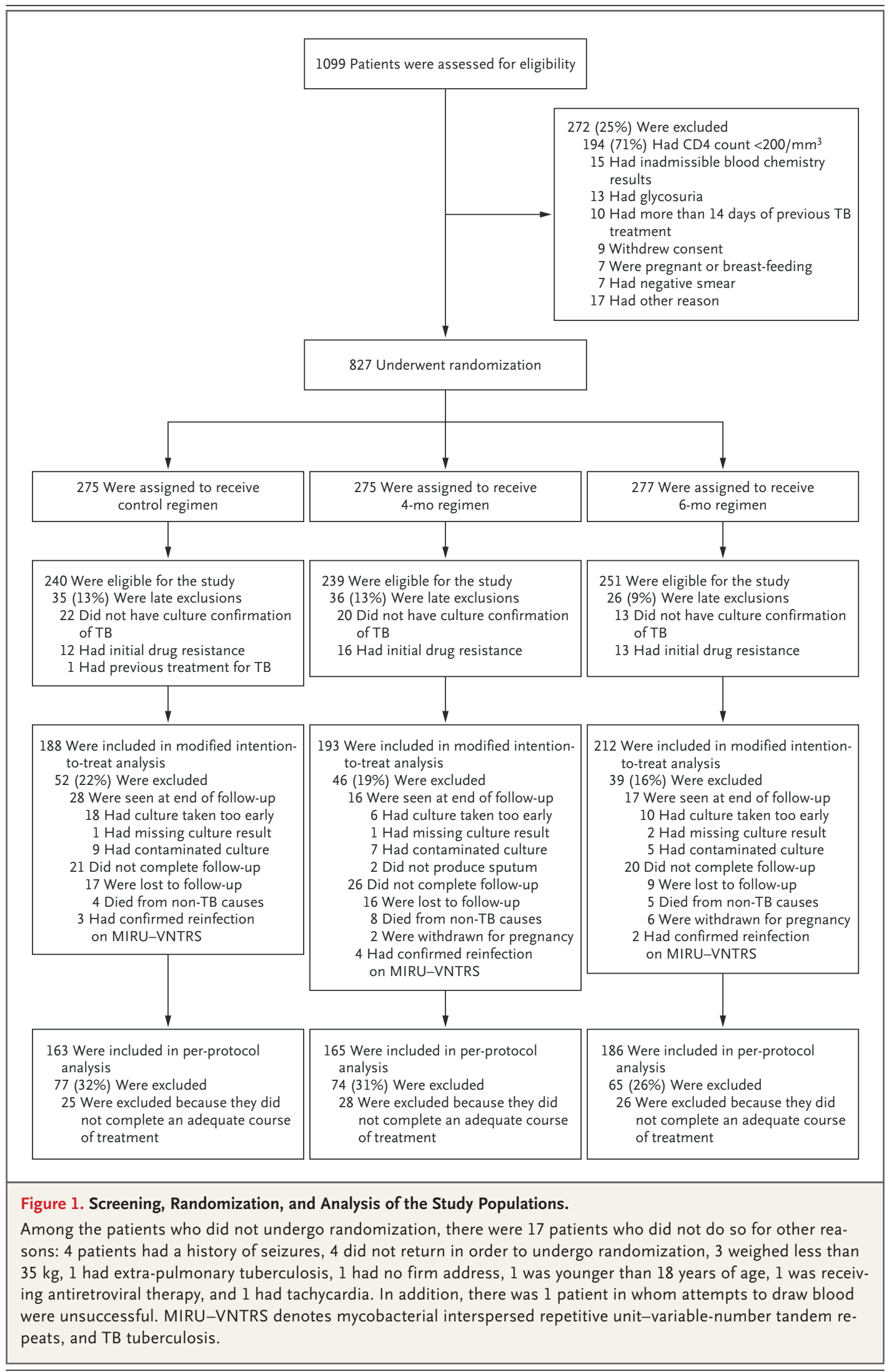

The New England Journal of Medicine 


\begin{tabular}{|c|c|c|c|c|}
\hline Characteristic & $\begin{array}{l}\text { Control } \\
\text { Regimen } \\
(\mathrm{N}=188)\end{array}$ & $\begin{array}{l}\text { 4-Month } \\
\text { Regimen } \\
(N=193)\end{array}$ & $\begin{array}{l}\text { 6-Month } \\
\text { Regimen } \\
(\mathrm{N}=212)\end{array}$ & $\begin{array}{c}\text { Total } \\
(\mathrm{N}=593)\end{array}$ \\
\hline Male sex — no. (\%) & $121(64)$ & $121(63)$ & $137(65)$ & $379(64)$ \\
\hline HIV-positive - no. (\%) & $54(29)$ & $55(28)$ & $49(23)$ & $158(27)$ \\
\hline \multicolumn{5}{|l|}{ Age - no. (\%) } \\
\hline $18-34 \mathrm{yr}$ & $115(61)$ & $132(68)$ & $127(60)$ & $374(63)$ \\
\hline $35-54 \mathrm{yr}$ & $66(35)$ & $55(28)$ & $78(37)$ & $199(34)$ \\
\hline$\geq 55$ & $7(4)$ & $6(3)$ & $7(3)$ & $20(3)$ \\
\hline \multicolumn{5}{|l|}{ Weight — no. (\%) } \\
\hline$<40 \mathrm{~kg}$ & $9(5)$ & $8(4)$ & $8(4)$ & $25(4)$ \\
\hline $40-54$ kg & $103(55)$ & $100(52)$ & $120(57)$ & $323(54)$ \\
\hline $55-69 \mathrm{~kg}$ & $71(38)$ & $77(40)$ & $82(39)$ & $230(39)$ \\
\hline$\geq 70 \mathrm{~kg}$ & $5(3)$ & $8(4)$ & $2(1)$ & $15(3)$ \\
\hline \multicolumn{5}{|c|}{ Smoking status - no. (\%) } \\
\hline Former smoker & $47(25)$ & $46(24)$ & $47(22)$ & $140(24)$ \\
\hline Current smoker & $46(24)$ & $51(26)$ & $64(30)$ & $161(27)$ \\
\hline Never smoked & $95(51)$ & $96(50)$ & $101(48)$ & $292(49)$ \\
\hline \multicolumn{5}{|l|}{ Cavitation - no. $(\%) *$} \\
\hline None & $57(33)$ & $64(35)$ & $73(37)$ & $194(35)$ \\
\hline $1-5 \mathrm{~cm}$ & $86(50)$ & $85(47)$ & $91(46)$ & $262(48)$ \\
\hline$>5 \mathrm{~cm}$ & $30(17)$ & $32(18)$ & $33(17)$ & $95(17)$ \\
\hline \multicolumn{5}{|l|}{ CD4 cell count $†$} \\
\hline Median & 355 & 317 & 298 & 314 \\
\hline Interquartile range & $247-455$ & $256-427$ & $247-383$ & $253-441$ \\
\hline \multicolumn{5}{|c|}{$\begin{array}{l}\text { Days of previous tuberculosis treatment } \\
\text { at randomization - no. }\end{array}$} \\
\hline Median & 5 & 4 & 4 & 4 \\
\hline Interquartile range & $1-8$ & $1-8$ & $1-7$ & $1-8$ \\
\hline
\end{tabular}

* Radiographs obtained at baseline were not available for 42 patients. Between-group baseline characteristics were compared by means of a chi-square test for independence for categorical variables and a nonparametric K-sample test of the equality of the medians for continuous variables. There were no statistically significant between-group differences in any of these baseline characteristics among the three treatment groups $(P>0.1)$.

$\dagger \mathrm{CD} 4$ cell counts are based on counts from 158 patients coinfected with the human immunodeficiency virus (HIV).

for the per-protocol analysis. The results are summarized in Figure 2. Kaplan-Meier estimates of the time to unfavorable outcome analysis for the per-protocol population are shown in Figure 3. With the 4-month regimen, 24 of 30 unfavorable outcomes $(80.0 \%)$ occurred less than 6 months after the end of treatment, a finding that is consistent with that of other trials. ${ }^{23}$

There was no statistically significant interaction between HIV status and treatment regimen in the per-protocol analysis or the modified intention-to-treat analysis. The differences between regimens were consistent if all deaths or all reinfections were classified as unfavorable (see Table S1 in the Supplementary Appendix for these and other sensitivity analyses). A strict modified intention-to-treat analysis including all post-randomization exclusions except for those determined late in screening indicated an increased benefit for patients receiving the 6-month regimen. How- 


\begin{tabular}{|c|c|c|c|c|}
\hline Status and Outcome & $\begin{array}{l}\text { Control } \\
\text { Regimen }\end{array}$ & $\begin{array}{l}\text { 4-Month } \\
\text { Regimen }\end{array}$ & $\begin{array}{l}\text { 6-Month } \\
\text { Regimen }\end{array}$ & Total \\
\hline Per-protocol analysis — no. & 163 & 165 & 186 & 514 \\
\hline Favorable - no. (\%) & $155(95.1)$ & $135(81.8)$ & $180(96.8)$ & $470(91.4)$ \\
\hline \multicolumn{5}{|l|}{ Unfavorable } \\
\hline Failure (culture confirmed) - no. & 2 & 2 & 0 & 4 \\
\hline Death during treatment — no. & 1 & 0 & 1 & 2 \\
\hline Relapse (culture confirmed) — no. & 4 & 19 & 4 & 27 \\
\hline Relapse (limited bacteriology) - no. & 1 & 7 & 1 & 9 \\
\hline Culture positive when last seen - no. & 0 & 2 & 0 & 2 \\
\hline Total — no. (\%) & $8(4.9)$ & $30(18.2)$ & $6(3.2)$ & $44(8.6)$ \\
\hline $\begin{array}{l}\text { Difference from control in unfavorable rate } \\
\text { (adjusted for study center) }\end{array}$ & & 13.6 & -1.8 & \\
\hline $90 \% \mathrm{Cl}$ & & 8.1 to 19.1 & -6.1 to 2.4 & \\
\hline $95 \% \mathrm{Cl}$ & & 7.0 to 20.2 & -6.9 to 3.3 & \\
\hline Modified intention-to-treat analysis — no. & 188 & 193 & 212 & 593 \\
\hline Favorable - no. (\%) & $161(85.6)$ & $141(73.1)$ & $183(86.3)$ & $485(81.8)$ \\
\hline \multicolumn{5}{|l|}{ Unfavorable } \\
\hline \multicolumn{5}{|l|}{ During treatment - no. } \\
\hline Failure (culture confirmed) & 2 & 2 & 0 & 4 \\
\hline Death & 1 & 0 & 1 & 2 \\
\hline $\begin{array}{l}\text { Change in treatment due to adverse } \\
\text { event }\end{array}$ & 1 & 2 & 2 & 5 \\
\hline Lost to follow-up & 5 & 6 & 8 & 19 \\
\hline Inadequate treatment & 2 & 1 & 3 & 6 \\
\hline Other treatment change $†$ & 10 & 11 & 10 & 31 \\
\hline \multicolumn{5}{|l|}{ After treatment - no. } \\
\hline \multicolumn{5}{|l|}{ Relapse } \\
\hline Culture confirmation & 4 & 19 & 4 & 27 \\
\hline $\begin{array}{l}\text { Limited bacteriologic confirma- } \\
\text { tion } \downarrow\end{array}$ & 2 & 8 & 1 & 11 \\
\hline Death due to tuberculosis & 0 & 1 & 0 & 1 \\
\hline Culture positive when last seen & 0 & 2 & 0 & 2 \\
\hline Total — no. (\%) & $27(14.4)$ & $52(26.9)$ & $29(13.7)$ & $108(18.2)$ \\
\hline $\begin{array}{l}\text { Difference from control in unfavorable rate } \\
\text { (adjusted for study center) }\end{array}$ & & 13.1 & 0.4 & \\
\hline $90 \% \mathrm{Cl}$ & & (6.8 to 19.4$)$ & $(-4.7$ to 5.6$)$ & \\
\hline $95 \% \mathrm{Cl}$ & & (5.6 to 20.6$)$ & $(-5.7$ to 6.6$)$ & \\
\hline
\end{tabular}

$* \mathrm{Cl}$ denotes confidence interval.

$\dagger$ Reasons for other treatment changes included poor adherence, withdrawal due to pregnancy after a positive culture, withdrawal of consent, and a move away from treatment center.

$\leftarrow$ Limited bacteriologic confirmation indicates that retreatment for relapse was initiated without confirmation on two positive cultures within 4 months after completion of treatment when no negative culture was obtained during the intervening 4 months. 


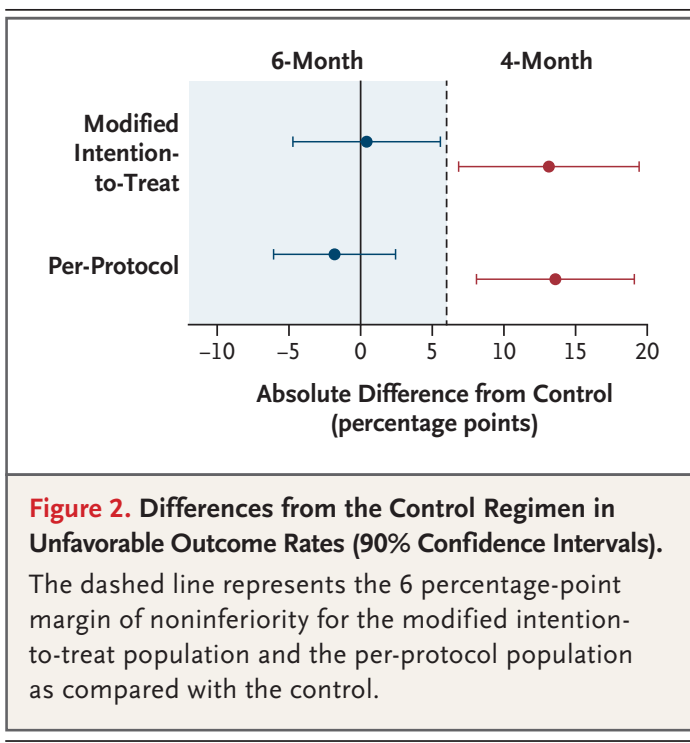

ever, this finding should be interpreted with caution, since fewer potential participants were excluded from this group, probably due to chance. Among 219 patients assessed at 2 months who received isoniazid for the first 2 months, 187 (85.3\%) had a negative culture as compared with 394 of 436 patients $(90.4 \%)$ who received moxifloxacin for the first 2 months $(\mathrm{P}=0.06)$ (Table S2 in the Supplementary Appendix).

Adherence to all three regimens was similar during the first 2 months of treatment. Thereafter, the proportions of patients with rates of adherence that were $89 \%$ or higher were similar in all three groups $(75.3 \%$ of patients receiving the control regimen, $81.4 \%$ of patients receiving the 4 -month regimen, and $80.9 \%$ of those receiving the 6-month regimen), but a smaller proportion of patients receiving the control regimen had adherence rates of $95 \%$ or higher $(48.7 \%$, as compared with $76.7 \%$ of those receiving the 4 -month regimen and $76.9 \%$ of those receiving the 6 -month regimen). The only instance of acquired resistance to rifampicin occurred in a patient in the control group who was coinfected with HIV and did not adhere to the control regimen.

\section{SAFETY}

A total of 45 adverse events graded as severe or life threatening occurred in 38 patients during treatment (Table S3 in the Supplementary Appendix), none of which were considered to have a definite relation to the study medication. However, 6 of the events in the control group, 6 of the events in the 4-month group, and 4 of the events in the 6-month group were considered to be possibly or probably related to the study medication. Among the 827 patients who underwent randomization, 25 patients died: 6 in the control group, 12 in the group receiving the 4-month regimen, and 7 in the group receiving the 6-month regimen. Among these 25 deaths, 4 were classified as being possibly or probably related to tuberculosis (see details in Table S4 in the Supplementary Appendix). Among the 8 patients for whom the cause of death could not be ascertained, all had negative results on the last sputum specimen that was cultured. However, 6 of these patients were coinfected with HIV, and their deaths could have been HIV-related.

DISCUSSION

This trial showed that a 6-month regimen with once-weekly dosing for the last 4 months was noninferior to the control regimen; no concerns related to safety were identified. In contrast, the 4-month regimen, in which moxifloxacin and $900 \mathrm{mg}$ of rifapentine were administered twice weekly during the continuation phase, had a significantly higher relapse rate than the control regimen. The selection of a $900-\mathrm{mg}$ dose of rifapentine for this regimen was based on the pharmacokinetic-pharmacodynamic assumptions that the bactericidal effect was proportional to the area under the concentration curve (AUC), which suggested that a regimen of $900 \mathrm{mg}$ of rifapentine twice weekly would be more effective than a regimen of $1200 \mathrm{mg}$ of rifapentine once weekly, and that the lower dose might reduce toxicity. Whereas studies in mice suggested that regimens shorter than the standard random were effective, this was not the case in our study. ${ }^{8,24}$ This discrepancy may be due to the presence of persisting subpopulations of bacilli that are in a dormant state in the lesions of pulmonary tuberculosis. These subpopulations of bacilli are thought to lead to relapse in humans, ${ }^{25}$ but they are largely absent in mice with experimentally induced tuberculosis. Because of these persisting subpopulations, bactericidal efficacy may be related to peak rifapentine concentrations in human lesions but related to the AUC in the mice. ${ }^{26}$ Consequently, the administration of $900 \mathrm{mg}$ of rifapentine twice weekly may not be more effective than the administration of $1200 \mathrm{mg}$ once weekly when 
treating tuberculosis in humans, even when these regimens are of the same duration.

The substitution of moxifloxacin for isoniazid was proposed after a report of an acceleration in bacillary elimination after such a substitution was made in a study in mice. ${ }^{7}$ In our trial, this substitution reduced the proportion of patients with positive cultures at 2 months from $14.6 \%$ to $9.6 \%$. This reduction compares with a decrease from $45 \%$ to $40 \%$ in a phase 2 trial evaluating the same moxifloxacin substitution, albeit with a slightly different definition of end point. ${ }^{27}$ However, the exact role of moxifloxacin in helping to prevent relapse is uncertain. In a nested pharmacokinetic study, the results showed that although rifapentine increased the clearance of moxifloxacin by $8 \%$ during treatment as compared with clearance after treatment completion without rifapentine, it did not result in a clinically significant change in moxifloxacin exposure. ${ }^{28}$ More work is needed to determine whether the substitution of moxifloxacin for isoniazid was a necessary component of the success of the once-weekly regimen of the continuation phase.

No acquired drug resistance was identified among patients receiving either of the intermittent regimens, but the numbers were too small to provide any definitive conclusion. Nevertheless, the low relapse rate associated with the 6-month regimen suggests that acquired resistance is unlikely to limit the usefulness of intermittent high dosing with rifapentine.

The trial had some limitations. The number of participants who underwent randomization (827) fell short of the target sample size (1095) due to a delayed start and slower-than-expected recruitment. However, the 827 participants were sufficient to interpret the results of both experimental regimens. Only $27 \%$ of patients included in the modified intention-to-treat analysis were coinfected with HIV; their median CD4 cell count was 314 per cubic millimeter. ${ }^{3}$ Patients coinfected with HIV were therefore underrepresented, particularly those with low CD4 counts. Although the ingestion of drugs during the continuation phase in the control group was supervised by a person other than clinic staff, predefined requirements for adequate adherence to treatment did not differ according to treatment regimen.

Expanding the adoption of rapid tests such as the Xpert MTB/RIF to exclude rifampicin-resistant disease would facilitate the safe use of the

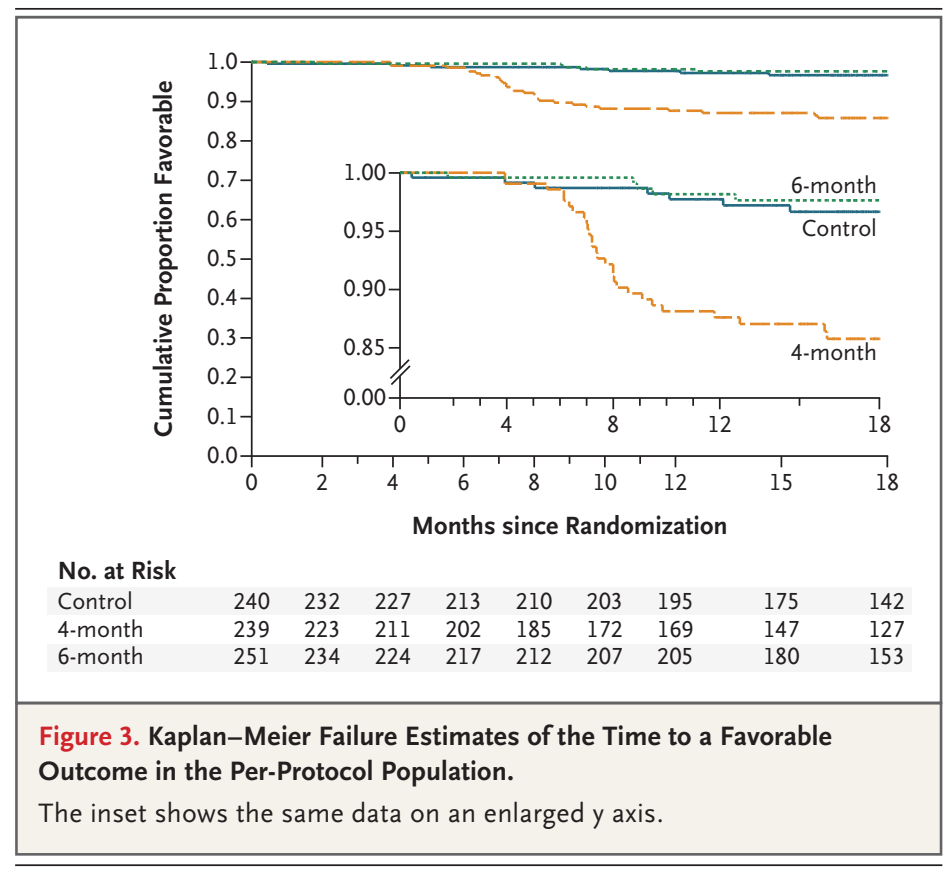

6-month regimen in resource-limited settings, making it possible to administer the regimen to patients with fully sensitive or mono-isoniazidresistant tuberculosis. The regimen is also particularly relevant for programs that rely on direct observation of daily treatment at health centers, since direct observation would be required only once weekly during the continuation phase and could therefore be delivered at a convenient location. Replacing the current daily treatment observation required by the control regimen with a regimen in which the intensity of treatment observation is reduced could lower some health-system costs, although the current costs of the drugs used in regimens requiring less observation are somewhat greater. No formal cost-effectiveness analysis was undertaken. However, Bayer has made a commitment to making moxifloxacin available at an affordable price, ${ }^{29}$ and recently Sanofi substantially reduced the cost of rifapentine in the United States. ${ }^{30}$ The meal of two boiled eggs and slices of bread provided to increase the absorption of rifapentine could be a barrier to implementation; further research is needed to determine to what extent this meal is necessary.

The 6-month regimen in which rifapentine and moxifloxacin are administered once weekly during the continuation phase is noninferior to the standard regimen, which requires daily administration of medication for 6 months. The new 
regimen could facilitate the strategy of directly observed treatment and could be used as firstline treatment in certain settings, such as those with low rates of HIV coinfection or high rates of isoniazid resistance.

Supported by the European and Developing Countries Clinical Trials Partnership, which provided funding for the trial, and the Wellcome Trust, which provided funding for the pharmacokinetic study.
No potential conflict of interest relevant to this article was reported.

Disclosure forms provided by the authors are available with the full text of this article at NEJM.org.

We thank Sanofi for the donation of rifapentine, rifampicin and isoniazid; Genus Pharmaceuticals for the donation of ethambutol; Sandoz for the donation of pyrazinamide; the members of the steering committee (Drs. Peter Godfrey-Faussett [chair], Gerry Davies, David Moore, and Oumou Bah-Sow); and the members of the data and safety monitoring committee (Drs. Tim Peto [chair], Alwyn Mwinga, and Katherine Fielding).
REFERENCES

1. Jindani A, Nunn AJ, Enarson DA. Two 8-month regimens of chemotherapy for treatment of newly diagnosed pulmonary tuberculosis: international multicentre randomised trial. Lancet 2004;364:124451.

2. Fox W, Ellard GA, Mitchison DA. Studies on the treatment of tuberculosis undertaken by the British Medical Research Council tuberculosis units, 19461986 , with relevant subsequent publications. Int J Tuberc Lung Dis 1999;3:Suppl 2:S231-79.

3. Food and Drug Administration. Priftin (rifapentine) drug approval package. October 2000(http://www.accessdata.fda .gov/drugsatfda_docs/nda/2000/21024S5_ Priftin.cfm).

4. Tam CM, Chan SL, Lam CW, et al. Rifapentine and isoniazid in the continuation phase of treating pulmonary tuberculosis; initial report. Am J Respir Crit Care Med 1998;157:1726-33.

5. Benator D, Bhattacharya M, Bozeman $\mathrm{L}$, et al. Rifapentine and isoniazid once a week versus rifampicin and isoniazid twice a week for treatment of drug-susceptible pulmonary tuberculosis in HIVnegative patients: a randomised clinical trial. Lancet 2002;360:528-34.

6. Vernon A, Burman W, Benator D, Khan A, Bozeman L. Acquired rifamycin monoresistance in patients with HIV-related tuberculosis treated with onceweekly rifapentine and isoniazid. Lancet 1999;353:1843-7.

7. Lounis N, Bentoucha A, Truffot-Pernot $\mathrm{C}$, et al. Effectiveness of once-weekly rifapentine and moxifloxacin regimens against Mycobacterium tuberculosis in mice. Antimicrob Agents Chemother 2001;45:3482-6.

8. Rosenthal IM, Williams K, Tyagi S, et al. Potent twice-weekly rifapentine-containing regimens in murine tuberculosis. Am J Respir Crit Care Med 2006;174:94101.

9. Den Boon S, Bateman ED, Enarson DA, et al. Development and evaluation of a new chest radiograph reading and recording system for epidemiological sur- veys of tuberculosis and lung disease. Int J Tuberc Lung Dis 2005;9:1088-96.

10. Dawson R, Masuka P, Edwards DJ, et al. Chest radiograph reading and recording system: evaluation for tuberculosis screening in patients with advanced HIV. Int J Tuberc Lung Dis 2010;14:52-8.

11. Treatment of tuberculosis: guidelines. 4th ed. Geneva: World Health Organization, 2010.

12. Zvada SP, Van Der Walt JS, Smith PJ, et al. Effects of four different meal types on the population pharmacokinetics of single-dose rifapentine in healthy male volunteers. Antimicrob Agents Chemother 2010;54:3390-4.

13. Division of AIDS. Table for grading severity of adult adverse experiences. Bethesda, MD: National Institute of Allergy and Infectious Diseases, 1992.

14. Lienhardt C, Cook SV, Burgos M, et al. Efficacy and safety of a 4-drug fixed-dose combination regimen compared with separate drugs for treatment of pulmonary tuberculosis: the Study C randomized controlled trial. JAMA 2011;305: 1415-23.

15. Merle CS, Sismanidis C, Sow OB, et al. A pivotal registration phase III, multicenter, randomized tuberculosis controlled trial: design issues and lessons learnt from the Gatifloxacin for $\mathrm{TB}$ (OFLOTUB) project. Trials 2012;13:61.

16. REMoxTB laboratory manual. June 2011 (http://www.ucl.ac.uk/infection-immunity/research/res_ccm/ccm_files/

CCM_REMox).

17. International Conference on Harmonisation of Technical Requirements for Registration of Pharmaceuticals for Human Use. Statistical principles for clinical trials (E9). 1998.

18. Machin D. Sample size tables for clinical studies. Chichester, United Kingdom: Wiley-Blackwell, 2009.

19. Proschan MA, Waclawiw MA. Practical guidelines for multiplicity adjustment in clinical trials. Control Clin Trials 2000; 21:527-39.

20. Day JN, Chau TT, Wolbers $M$, et al. Combination antifungal therapy for cryp- tococcal meningitis. N Engl J Med 2013; 368:1291-302.

21. Schulz KF, Grimes DA. Multiplicity in randomised trials I: endpoints and treatments. Lancet 2005;365:1591-5.

22. Schulz KF, Grimes DA. Multiplicity in randomised trials II: subgroup and interim analyses. Lancet 2005;365:1657-61.

23. Nunn AJ, Phillips PPI, Mitchison DA. Timing of relapse in short-course chemotherapy trials for tuberculosis. Int $\mathrm{J}$ Tuberc Lung Dis 2010;14:241-2.

24. Rosenthal IM, Zhang M, Williams $\mathrm{KN}$, et al. Daily dosing of rifapentine cures tuberculosis in three months or less in the murine model. PLoS Med 2007; 4(12):e344.

25. Garton NJ, Waddell SJ, Sherratt AL, et al. Cytological and transcript analyses reveal fat and lazy persister-like bacilli in tuberculous sputum. PLoS Med 2008;5(4): e75.

26. Mitchison DA. Pharmacokinetic/ pharmacodynamic parameters and the choice of high-dosage rifamycins. Int J Tuberc Lung Dis 2012;16:1186-9.

27. Dorman SE, Johnson JL, Goldberg S, et al. Substitution of moxifloxacin for isoniazid during intensive phase treatment of pulmonary tuberculosis. Am J Respir Crit Care Med 2009;180:273-80.

28. Zvada SP, Denti P, Geldenhuys H, et al. Moxifloxacin population pharmacokinetics in patients with pulmonary tuberculosis and the effect of intermittent high-dose rifapentine. Antimicrob Agents Chemother 2012;56:4471-3.

29. Bayer and TB Alliance to develop moxifloxacin for TB. Press release release of the TB Alliance, October 2005 (http:/l tballiance.org/newscenter/view-brief .php?id=158).

30. Tuberculosis drug maker Sanofi drops price, easing access to shortened $\mathrm{TB}$ infection treatment. Arlington, VA: Center for Global Health Policy. December 13, 2013 (http://sciencespeaksblog.org/ 2013/12/13/tb-drug-maker-drops-price -offering-hope-of-shortened-affordable -treatment).

Copyright (c) 2014 Massachusetts Medical Society. 